D.V. Tugay

\title{
THREE-PHASE ENERGY SUPPLY SYSTEMS SIMULATION FOR THE TOTAL POWER LOSSES COMPONENTS ASSESSMENT
}

Purpose. The goal is to optimize a structure of Matlab-model of the three-phase energy supply system with power active filter. The mathematical model that describes the energy supply system modes of operation which contains additional losses is proposed. Methodology. We have applied concepts of the electrical circuits theory, mathematical modeling elements based on linear algebra and vector calculus, mathematical simulation in Matlab package. Results. We have developed two models of three-phase energy supply system. The first one is based on a vector representation, and the second one on the matrix representation of energy processes. Using these models we have solved the problem of maintaining unchanged the average useful power for 279 cases of energy supply system modes of operation. Originality. We have developed methods of mathematical analysis of a three-phase energy supply systems with polyharmonic voltages and currents in the symmetric and asymmetric modes. Practical value. We have created Matlab-model of a three-phase energy supply system with automated calculation of a correction factor. It allows reducing more than one order the time for energy processes elucidation in multiphase systems. References 11, tables 1 , figures 13. Key words: energy supply system, power active filter, the minimum possible losses, total losses power, three-dimensional complex vector, Matlab-model of the three-phase energy supply system.

Цель. Целью статьи является оптимизация структуры Маtlab-модели трехфазной системы электроснабжения с силовым активным фильтром с помощььо математической модели, описывающей режимы работы системы электроснабжения, в которых возникают дополнительные потери. Методика. Для проведения исследований использовались положения теории электрических цепей, элементы математического моделирования, основанные на линейной алгебре и векторном исчислении, математическое моделирование в пакете Маtlab. Результаты. Разработаны две модели трехфазной системы электроснабжения, первая, основанная на векторном представлении, а вторая на матричном представлении энерzетических процессов, с помощью которых была решена проблема поддержания неизменной средней полезной мощности нагрузки для 279 случаев работы системы электроснабжения. Научная новизна. Получили дальнейшее развитие методы математического анализа режсимов работы трехфазных систем электроснабжения с полигармоническими напряжениями и токами в симметричных и несимметричных режнмах работы. Практическое значение. Создана Маtlab-модель трехфазной системы электроснабжения с автоматизированным расчетом поправочного коэффициента, позволяющая более чем на порядок сократить время для исследования энергетических процессов в многофазных системах. Библ. 11, рис. 13, табл. 1.

Ключевые слова: система электроснабжения, силовой активный фильтр, минимально возможные потери, мощность суммарных потерь, трехмерный комплексный вектор, Маtlab-модель трехфазной системы электроснабжения.

Introduction. Understanding the operation of threephase energy supply systems (ESS) allows professionals in the field of power electronics to solve a number of problems related to the electromagnetic compatibility of electric power consumers from the industrial network as well as increasing the energy efficiency of such systems. Complex solution of problems presented in the current conditions based on the use of the active filtering means power active filters (PAF). In the systems of energy supply of communal consumers, industrial plants, electric vehicles adaptation of PAF of parallel type is increased $[1,2]$. Despite the simplicity of the power circuit which is a three-phase voltage inverter on the transistor modules, PAF are complex dynamic systems able to adapt to changes in the configuration and mode of operation of three-phase ESS. The feasibility of using PAF to reduce the losses in the ESS [3, 4] is due to the possibility of a preliminary assessment of the total power losses in the system before and after it is connected. The simplest way to perform such an assessment is a computer simulation of a three-phase ESS with PAF. This paper describes how to create a computer model to investigate diversity of power modes of the three-phase ESS with minimal time overhead.

A complicated extensive three-phase ESS can be replaced by a simple equivalent circuit shown in Fig. $1[5]$.

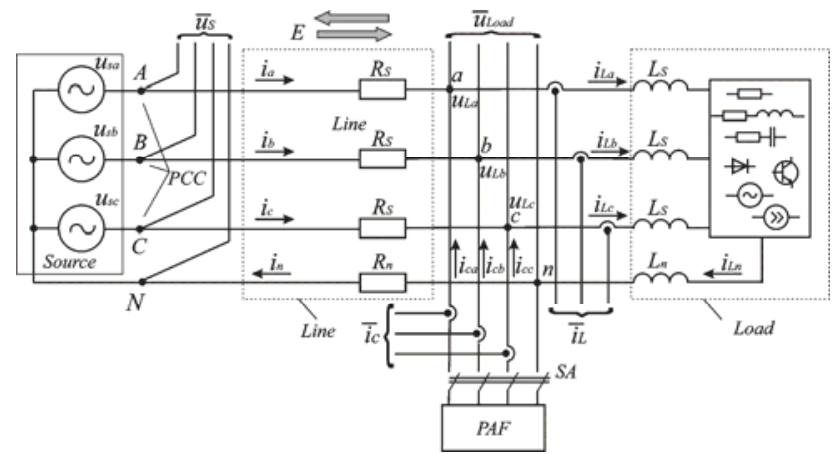

Fig. 1. Equivalent circuit of the three-phase ESS with PAF

The scheme consists of three parts: a three-phase sinusoidal power source of sinusoidal (or nearly sinusoidal) voltages Source, a three-phase load Load and connecting cable line Line with resistance linear wire $R_{s}$ and neutral wire resistance $R_{n}$. The three-phase load in the circuit of Fig. 1 may be any of: resistors, reactors, capacitors batteries, nonlinear consumers, current and voltage sources as well as the possible combinations of the elements listed. It should be noted that the inductances $L_{s}$ and $L_{n}$ of the cable line in the equivalent circuit are transferred in the load. Depending on the nature of the load and the operating mode of the ESS three power transmission modes are available: direct - energy is 
transmitted from the source to the load, reverse - the energy is transferred from the load to the source, and mixed mode - in the period of recurrence it is possible to combine the first and the second modes. After the contactor $S A$ switch on, the power active filter (block $P A F)$ is connected parallel to the load.

In [6] a universal formula to determine the total losses power in the three-phase four-wire ESS through its components is proposed

$$
\begin{aligned}
& \Delta P_{\Sigma^{*}}=\frac{\Delta P_{\Sigma}}{P_{u s f}}= \\
& =\Delta P_{\min *}+\Delta P_{p u l s^{*}}+\Delta P_{q^{*}}+\Delta P_{n^{*}}+\Delta P_{m u t^{*}}= \\
& =\Delta P_{\min *}+\left.\Delta P_{a d d^{*}}\right|_{P_{u s f}=\text { const },}
\end{aligned}
$$

where $\Delta P_{\text {min }} *$ is the relative minimum possible power losses determined by the absence of pulsations of the instantaneous active power and the vanishing of the instantaneous reactive power in three-phase ESS; $\Delta P_{p u l s}{ }^{*}$ is the relative components of additional power losses due to the transient component of the instantaneous power of the three-phase ESS; $\Delta P_{q^{*}}$ is the relative component of additional losses power due to the instantaneous reactive power of the three-phase ESS; $\Delta P_{n^{*}}$ is the relative power losses in the neutral conductor calculated in the period of repeatability caused by current flow in the neutral wire; $\Delta P_{m u t^{*}}$ is the relative component of additional losses power due to the mutual influence of the electromagnetic processes in the phase conductors and the neutral wire of the three-phase ESS; $\Delta P_{a d d^{*}}$ is the relative additional losses power; $P_{u s f}$ is the average calculated in the period of repeatability useful load power the amount of which in the course of the experiment must be kept constant.

Calculation of the total power losses components is based on the concepts of modern theories of instantaneous active and reactive power [7-9] on the nature of the electromagnetic processes in three-phase ESS and algorithms of PAF control using matrix transformations of these theories. In the simplest case, if we consider the ideal active filter (with zero internal losses) then when it is connected to the three-phase ESS total losses in the system will be equal to the lowest possible, i.e. the ESS will operate with the highest possible efficiency [10]

$$
\begin{gathered}
\eta_{\max }=\frac{1}{2}+\sqrt{\frac{1}{4}-\frac{1}{k_{s c}}}, \\
k_{s c}=P_{s c} / P_{u s f}
\end{gathered}
$$

is the ratio of power of the three-phase resistive short circuit of the ESS to useful load power.

In above-mentioned publications $[5,6]$ it was shown that the validation of (1) is possible using the Matlabmodel of three-phase four-wire ESS which has properties of universality and allows the calculation of power losses components at different modes of the ESS. Combinations of modes of three-phase voltage source, the nature of three-phase load, the neutral conductor resistance value and direction of the energy flow in the line wires allow to obtain 288 ESS variants, while in 279 ESS variants there are additional losses described by (1). The authors $[5,6]$ were able to check about 30 ESS variants, the investigation of which confirmed the validity of (1) but was associated with significant time-consuming.

This work is dedicated to the creation of a universal Matlab-model of the three-phase ESS and optimization of its parameters for an accelerated assessment of the power losses components in any of the 288 possible ESS variants.

The structure of the three-phase ESS model. Optimization of the structure of the Matlab-model of the three-phase ESS may be carried out at the transition from the circuit on Fig. 1 to the equivalent circuit of Fig. 2.

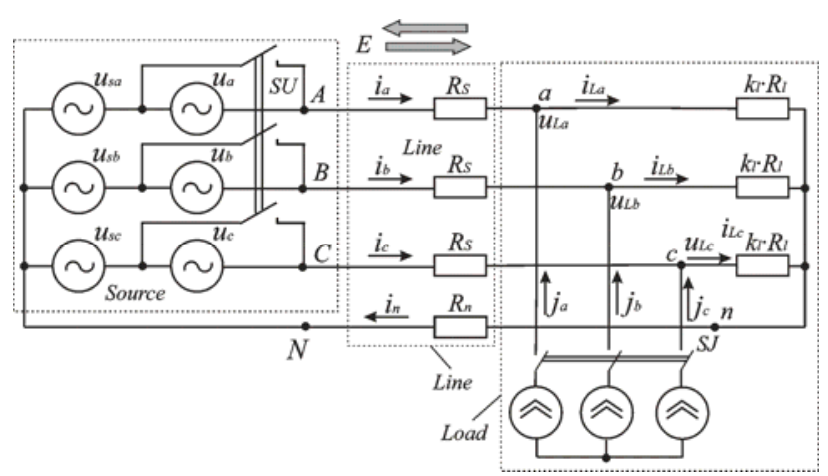

Fig. 2. Equivalent circuit of the three-phase ESS

The three-phase voltage source in this circuit consists of two series-connected sources - symmetrical sinusoidal voltage source $u_{s a}, u_{s b}, u_{s c}$ and additional threephase voltage source $u_{a}, u_{b}, u_{c}$ which is connected to the ESS by opening the bypass contactor $S U$. With the additional power source it is possible to specify amplitude or phase asymmetry as well as the addition of the higher harmonic components to the range of supply voltage. The three-phase load is modeled by two blocks - a symmetrical three-phase resistive load $k_{l} \cdot R_{l}$ and regulated three-phase current source $j_{a}, j_{b}, j_{c}$ which is connected in parallel with the symmetrical resistive load after $S J$ contactor closing. Regulated current source provides in linear conductors the necessary current shape that will fit any linear or non-linear load with balanced or unbalanced phases load. The proportionality factor $k_{l}$ which is multiplied by the active resistances of all three phases is necessary to maintain a constant value of average active useful power of load $P_{u s f}=$ const as follows from (1) when making a particular disturbance in the system, and its definition is a separate problem.

Setting variable coefficients that determine the operation mode of the three-phase ESS. We return to the ESS equivalent circuits on Fig. 1 when the contactor $S A$ is open and replace it with a simplified diagram shown in Fig. 3 explaining the cause of the power loss components in accordance with (1). Electrical quantities and parameters in the circuit of Fig. 3 are shown in the vector notation. The circuit consists of a voltage source the phase values of which are described by the vector $\vec{u}=\overrightarrow{k_{u}} \circ \overrightarrow{u_{s}}+\overrightarrow{u_{n s}} \circ \vec{k}_{u n} \cdot s_{u}$, resistance of the line $R_{S} \cdot \vec{v}$, complex linear load resistance for the $i$-th harmonic component of the current which is described by the vector $\overrightarrow{z_{L i}}=k_{l} \cdot R_{l} \cdot \overrightarrow{k_{a}}+j \cdot\left(x_{l i} \cdot \overrightarrow{k_{r}}+x_{s i}^{\prime} \cdot \vec{v}\right)$, and the 
current source modeling the nonlinear load described by the vector $\vec{k}_{j} \circ \vec{J} \cdot s_{j}$.

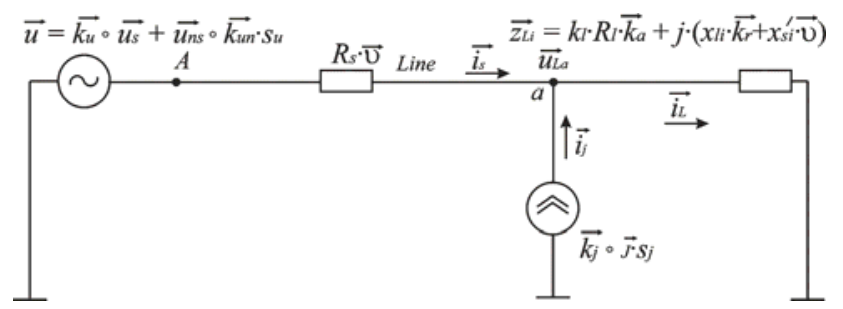

Fig. 3. Simplified circuit of the three-phase ESS for the parameters setting

In Fig. 3 the following notation is used:

- the 3D complex vector of phase symmetrical sinusoidal voltages

$$
\vec{u}_{S}=\left[\begin{array}{c}
U_{s} \cdot e^{j \cdot 0} \\
U_{s} \cdot e^{-j \cdot \frac{2 \pi}{3}} \\
U_{s} \cdot e^{-j \cdot \frac{4 \pi}{3}}
\end{array}\right]=\left[\begin{array}{l}
\underline{U}_{s a} \\
\underline{U}_{s b} \\
\underline{U}_{s c}
\end{array}\right],
$$

where $U_{s}$ is the RMS phase voltage of the symmetric source;

- the 3D complex vector of phase voltage of the additional three phase source of non-sinusoidal voltage

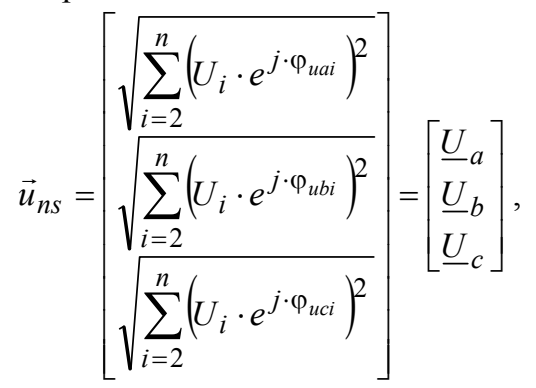

where $U_{i}$ is the RMS of the $i$-th harmonic component of phase voltage of three-phase non-symmetric source, $n$ is the number of higher harmonics in the voltage range; $\varphi_{\text {uai }}$, $\varphi_{u b i}, \varphi_{u c i}$ are the phases of corresponding $i$-th harmonic components of phase voltages; $s_{u}$ is the coefficient taking two values 0 or 1 depending on the switch $S U$ position in Fig. 2; $\circ$ is the operator of element-wise multiplication of elements of vectors and matrices (Hadamard product);

- the 3D complex vector of phase currents of the nonlinear load

$$
\vec{J}=\left[\begin{array}{l}
\sqrt{\sum_{i=2}^{n}\left(J_{i} \cdot e^{j \cdot \varphi_{i a i}}\right)^{2}} \\
\sqrt{\sum_{i=2}^{n}\left(J_{i} \cdot e^{j \cdot \varphi_{i b i}}\right)^{2}}
\end{array}=\left[\begin{array}{l}
\underline{J}_{a} \\
\underline{J}_{b} \\
\underline{J}_{c}
\end{array}\right],\right.
$$

where $J_{i}$ is the RMS of the $i$-th harmonic component of the phase current of the three-phase nonlinear load; $\varphi_{i a i}, \varphi_{i b i}, \varphi_{i c i}$ are the phases of corresponding $i$-th harmonic components of phase currents; $s_{j}$ is the coefficient taking two values 0 or 1 depending on the switch $S J$ position in Fig. 2;

- the 3D complex vector of linear load resistances

$$
\begin{aligned}
& \vec{z}_{L i}=\left[\begin{array}{l}
k_{l} \cdot R_{l} \cdot k_{a a}+j \cdot\left(x_{l i} \cdot k_{r a}+x_{s i}^{\prime}\right. \\
k_{l} \cdot R_{l} \cdot k_{a b}+j \cdot\left(\begin{array}{l}
x_{l i} \cdot k_{r b}+x_{s i}^{\prime} \\
k_{l} \cdot R_{l} \cdot k_{a c}+j \cdot\left(x_{l i} \cdot k_{r c}+x_{s i}^{\prime}\right.
\end{array}\right)
\end{array}\right]= \\
& =\left[\begin{array}{c}
Z_{L a i} \cdot e^{j \cdot \varphi_{z a i}} \\
Z_{L b i} \cdot e^{j \cdot \varphi_{z b i}} \\
Z_{L c i} \cdot e^{j \cdot \varphi_{z c i}}
\end{array}\right]=\left[\begin{array}{l}
\underline{Z}_{L a} \\
\underline{Z}_{L b} \\
\underline{Z}_{L c}
\end{array}\right],
\end{aligned}
$$

where $x_{l i}$ are the reactances for the $i$-th current harmonic; $x_{s i}^{\prime}$ is the reactance of the cable line for the $i$-th current harmonic reduced to the load reactance; $Z_{L a i}, Z_{L b i}, Z_{L c i}$ are the modules of the impedance of linear load phases for the $i$-th current harmonic; $\varphi_{z a}, \varphi_{z b}, \varphi_{z c}$ are the phases of corresponding impedances;

- the 3D vectors which store information about coefficients of amplitude asymmetry of the voltage source and non-linear load current, phase unbalance of active and reactive load as well as the value of the corresponding harmonic component of the input voltage range and a non-linear load current

$$
\begin{aligned}
\vec{k}_{u} & =\left[\begin{array}{lll}
k_{u a} & k_{u b} & k_{u c}
\end{array}\right]^{T}, \\
\vec{k}_{u n} & =\left[\begin{array}{lll}
k_{u n a} & k_{u n b} & k_{u n c}
\end{array}\right]^{T}, \\
\vec{k}_{j} & =\left[\begin{array}{lll}
k_{j a} & k_{j b} & k_{j c}
\end{array}\right]^{T}, \\
\vec{k}_{a} & =\left[\begin{array}{lll}
k_{a a} & k_{a b} & k_{a c}
\end{array}\right]^{T}, \\
\vec{k}_{r} & =\left[\begin{array}{lll}
k_{r a} & k_{r b} & k_{r c}
\end{array}\right]^{T} ;
\end{aligned}
$$

- the unit vector

$$
\vec{v}=\left[\begin{array}{lll}
1 & 1 & 1
\end{array}\right]^{T}
$$

By setting the values of $s_{u}, s_{j}$ factors and elements of vectors (8) - (12) it is possible to simulate any of 279 modes of operation of the three-phase ESS and calculate the components of the total power losses. To summarize the results of the calculations we assume that the values of the elements of the vectors in (8), (11) are linked by a common regularity

$$
k_{a}^{2}+k_{b}^{2}+k_{c}^{2}=3 .
$$

The coefficient of the phase A $k_{a}$ remains unchanged and equal to 1 , then

$$
\begin{aligned}
& k_{a}=\text { const }=1 \\
& k_{b}=\operatorname{var}=0 \ldots \sqrt{2} \\
& k_{c}=\sqrt{2-k_{b}^{2}}=\sqrt{2} \ldots 0
\end{aligned} .
$$

We assume that the values of the elements of the vectors in (9), (10), (12) subject to other regularity

$$
\begin{aligned}
& k_{b}=\operatorname{var}=0 \ldots \sqrt{2} \\
& k_{a}=k_{c}=k_{b}
\end{aligned}
$$

Regularities of change of vector elements values (15) and (16) will be used later to summarize the simulation results for different operation modes of threephase ESS. Note that these ratios may vary in any arbitrary manner, and the selected regularities (15) and (16) represent the most characteristic changes influences of the three-phase ESS parameters on the total losses. 
Fulfillment of the condition $\boldsymbol{P}_{u s f}=$ const at the three-phase energy supply system modeling.

Model representation in the vector form. Changes in the parameters of the elements of the equivalent circuit of three-phase ESS changes the value of the average active useful load power. Returning its initial value, as previously mentioned, is carried out by varying the coefficient $k_{l}$. Obtaining the analytical expressions for determination of $k_{l}$ is a difficult problem. It is easier to use the software for calculations. For automated calculation of the coefficient $k_{l}$ it is necessary to build the equation for the useful load power as shown in Fig. 3. There are two forms of this equation - in the vector and matrix form. We consider the first method.

We use the method of the current superposition. In the circuit of Fig. 3 we leave the voltage source. We define the vector of three-phase ESS conductivities for the $i$-th current harmonic

$$
\vec{y}_{i}=\frac{1}{\vec{z}_{L i}+R_{S} \cdot \vec{v}} .
$$

The total conductivity of the three-phase four-wire ESS for the $i$-th current harmonic

$$
y_{s i}=\vec{y}_{i} \cdot \vec{v}+R_{n}^{-1} .
$$

We write the dependence for the neutral voltage displacement for the main harmonic of the three-phase voltage source

$$
\underline{U}_{01}=\vec{y}_{1} \cdot y_{s 1}^{-1} \cdot\left(\vec{u}_{s} \circ \vec{k}_{u}\right) .
$$

Vector of partial currents created by main voltage harmonic of the linear source in the ESS linear wires

$$
\vec{i}_{e 1}=\left(\vec{u}_{s}-\underline{U}_{01} \cdot \vec{v}\right) \circ \vec{y}_{1} .
$$

If the higher harmonics (coefficient $s_{u}=1$ ) are in the input voltage curve, the procedure for calculating the vectors of the partial currents created by the three-phase voltage source must be repeated for each harmonic component using (7), (17) - (20). The total vector of the partial currents created by the three-phase voltage source in the ESS line wires in this mode is described by the relation

$$
\vec{i}_{e}=\sqrt{\left(\vec{i}_{e 1}\right)^{2}+\sum_{i=2}^{n}\left(\vec{i}_{e i}\right)^{2}},
$$

where $\vec{i}_{e i}$ is the partial current vector of the $i$-th harmonic component in the circuit with three-phase voltage source.

In the circuit of Fig. 3 we leave the current source pre-shorting the voltage source, and define the vector of partial linear load current in the circuit with three-phase current source

$$
\begin{gathered}
\vec{i}_{L j}=\left[\begin{array}{l}
\sqrt{\sum_{i=2}^{n}\left(J_{i} \cdot Y_{a i} \cdot R_{S} \cdot e^{j \cdot\left(\varphi_{i a i}+\varphi_{y a i}\right)}\right)^{2}} \\
\sqrt{\sum_{i=2}^{n}\left(J_{i} \cdot Y_{b i} \cdot R_{S} \cdot e^{j \cdot\left(\varphi_{i b i}+\varphi_{y b i}\right)}\right)^{2}} \\
\qquad \sqrt{\sum_{i=2}^{n}\left(J_{i} \cdot Y_{c i} \cdot R_{s} \cdot e^{j \cdot\left(\varphi_{i c i}+\varphi_{y c i}\right)}\right)^{2}}
\end{array},\right. \\
\circ \vec{k}_{j}=\sqrt{\sum_{i=2}^{n}\left(\vec{i}_{L j i}\right)^{2}}=\left[\begin{array}{c}
\underline{I}_{a L j} \\
\underline{I} b L j \\
\underline{I} c L j
\end{array}\right]
\end{gathered}
$$

where $Y_{a i}, Y_{b i}, Y_{c i}$ are the modules of total load phase conductivities for the $i$-th current harmonics; $\varphi_{y a i}, \varphi_{y b i}, \varphi_{y c i}$ are the initial phases of corresponding conductivities.

We find the vector of partial current created by the three-phase current source in ESS linear wires by the first Kirchhoff law

$$
\vec{i}_{s j}=\vec{J}-\vec{i}_{L j} .
$$

We determine the current vector in the linear load circuit

$$
\vec{i}_{L}=\sqrt{\left(\vec{i}_{e}\right)^{2}+\left(\vec{i}_{L j}\right)^{2}},
$$

and find the voltage vector on the load terminals

$$
\vec{u}_{L}=\sqrt{\left(\vec{i}_{e 1} \circ \vec{z}_{L 1}\right)^{2}+\sum_{i=2}^{n}\left(\vec{i}_{e i} \circ \vec{z}_{L i}\right)^{2}+\sum_{i=2}^{n}\left(\vec{i}_{L j i} \circ \vec{z}_{L i}\right)^{2}} .
$$

Average active useful power

$$
\begin{aligned}
P_{u s f} & =\operatorname{Re}\left(\vec{u}_{L} \cdot \sqrt{\left(\vec{i}_{e}\right)^{2}+\left(\vec{i}_{s j}\right)^{2}-(J)^{2}}\right)= \\
& =\operatorname{Re}\left(\vec{u}_{L} \cdot \sqrt{\left(\vec{i}_{L}\right)^{2}-2 \cdot\left(\vec{J} \circ \vec{i}_{L j}\right)}\right)
\end{aligned}
$$

Model representation in the matrix form. Vector form for three-phase models of ESS has some disadvantage manifests itself at polyharmonic shape of the supply voltage. With this it is difficult to describe the mutual influence of the higher voltage harmonic components of the three-phase source and higher current harmonics flowing in the non-linear load phases. In such a case it is convenient to use the matrix form of the model description.

Let's set the voltage at the three-phase source terminal by the matrix size $n \times 3$

$$
\mathbf{u}=\left\|\begin{array}{ccc}
\underline{U}_{a 1} & \underline{U}_{b 1} & \underline{U}_{c 1} \\
\vdots & \vdots & \vdots \\
\underline{U}_{a n} & \underline{U}_{b n} & \underline{U}_{c n}
\end{array}\right\| .
$$

Similarly, we write the matrix of the three-phase current source

$$
\mathbf{j}=\left\|\begin{array}{|ccc}
\underline{J}_{a 2} & J_{b 2} & J_{c 2} \\
\vdots & \vdots & \vdots \\
\underline{J}_{a n} & \underline{J}_{b n} & \underline{J}_{c n}
\end{array}\right\| .
$$

Separately, we define a matrix of complex load impedances for the three-phase voltage source currents and three-phase current source 


$$
\begin{aligned}
& \mathbf{z}_{\mathbf{u}}=\left\|\begin{array}{ccc}
\underline{z}_{a u 1} & \underline{z}_{b u 1} & \underline{z}_{c u 1} \\
\vdots & \vdots & \vdots \\
\underline{z}_{\text {aun }} & \underline{z}_{\text {bun }} & \underline{z}_{c u n}
\end{array}\right\|, \\
& \mathbf{z}_{\mathbf{j}}=\left\|\begin{array}{ccc}
\underline{z}_{a j 2} & \underline{z}_{b j 2} & \underline{z}_{c j 2} \\
\vdots & \vdots & \vdots \\
\underline{z}_{a j n} & \underline{z}_{b j n} & \underline{z}_{c j n}
\end{array}\right\| .
\end{aligned}
$$

We express the complex matrix of phase conductivities for three-phase voltage source currents

$$
\mathbf{y}_{\mathbf{u}}=\frac{\mathbf{M}_{\mathbf{u}}}{\mathbf{z}_{\mathbf{u}}+R_{S}},
$$

where $\mathbf{M}_{\mathbf{u}}$ is the matrix of states size $n \times 3$ whose rows are taking the unit values in the case of presence of corresponding harmonic components in the range of the three-phase voltage source.

We write the vector of total complex impedances of the three-phase four-wire system

$$
\vec{y}_{s}=\mathbf{y}_{\mathbf{u}} \cdot \vec{v}+R_{n}^{-1} .
$$

We represent the current in the neutral wire due to the higher harmonic components multiple three of the three-phase current source, as a vector of the length $n$

$$
\vec{i}_{3 j}=\mathbf{j} \circ \frac{\mathbf{z}_{\mathbf{j}}}{R s+3 \cdot R_{n}+\mathbf{z}_{\mathbf{j}}} \cdot \vec{v} .
$$

The displacement voltage at the neutral at polyharmonic supply voltage and polyharmonic current is represented as a vector of the length $n$

$$
\vec{u}_{0}=\frac{\mathbf{y}_{\mathbf{u}} \circ \mathbf{u}}{\vec{y}_{s}} \cdot \vec{v}-\vec{i}_{3 j} \cdot R_{n} .
$$

The line currents matrix in the circuit with threephase voltage source

$$
\mathbf{i}_{\mathbf{e}}=\left(\mathbf{u}-\vec{u}_{0} \cdot \vec{v}^{T}\right) \circ \mathbf{y}_{\mathbf{u}} .
$$

Using matrix relations (28) and (30) we express the partial currents of the three-phase current source, respectively, in the load and linear wires

$$
\begin{aligned}
\mathbf{i}_{\mathbf{L j}} & =\mathbf{j} \circ \frac{R s}{R s+\mathbf{z}_{\mathbf{j}}}, \\
\mathbf{i}_{\mathbf{s} \mathbf{j}} & =\mathbf{j}-\mathbf{i}_{\mathbf{L} \mathbf{j}} .
\end{aligned}
$$

We determine total line current and total current in the linear load phases

$$
\begin{aligned}
& \mathbf{i}_{\mathbf{s}}=\mathbf{i}_{\mathbf{e}}-\mathbf{i}_{\mathbf{s j}}, \\
& \mathbf{i}_{\mathbf{L}}=\mathbf{i}_{\mathbf{e}}+\mathbf{i}_{\mathbf{L j}}
\end{aligned}
$$

And find the voltage on the load terminals

$$
\mathbf{u}_{\mathbf{L}}=\mathbf{i}_{\mathbf{e}} \circ \mathbf{z}_{\mathbf{u}}+\mathbf{i}_{\mathbf{L j}} \circ \mathbf{z}_{\mathbf{j}} .
$$

Average active useful load power

$$
P_{u s f}=\left(\left|\mathbf{u}_{\mathbf{L}}\right| \circ\left(\left|\mathbf{i}_{\mathbf{L}}\right|-|\mathbf{j}|\right)\right)^{T} \cdot \mathbf{M}_{\mathbf{u}}^{\langle\mathbf{1}\rangle} \cdot \vec{v} .
$$

Both described models allow to relatively simply calculate the value of the correction factor $k_{l}$ returning the original value of the average load useful power even when using standard MathCad tools (Given block and Find function). After that it is possible to determine components of the power losses and the comparison of calculation results at ESS different modes of operation.

As an example, we consider a three-phase ESS with a ratio of three-phase power of resistive short circuit to load active power $k_{s c}=20$. The RMS of the phase sinusoidal voltage of the three-phase symmetric source $U_{s}$ $=220 \mathrm{~V}$. If the useful load power at the mode of minimum of losses equal to $P_{u s f}=400.1 \mathrm{~kW}$, then $R_{s}=$ $0.01815 \Omega, R_{l}=0.3256 \Omega$. According to (8) - (12) we consider separately 5 modes of ESS operation, each of which is caused by one factor influencing the occurrence of additional losses:

1. Voltage unbalance.

2. Resistive load unbalance.

3. Symmetric active-inductive load at $\varphi_{L}=15^{\circ}(L=$ $=0.2777 \mathrm{mH}$ ).

4. Higher harmonics in the supply voltage curve (odd harmonics whose amplitudes vary inversely their serial number $U_{m} / n$, where $\left.n=2 i-1, i=2,3 \ldots 19\right)$.

5 . The symmetrical non-linear load resulting in the higher current harmonics (odd harmonics, the amplitudes of which vary inversely their serial number $U_{m} /\left(n \cdot\left(R_{s}+R_{l}\right)\right)$, where $\left.n=2 i-1, i=2,3 \ldots 19\right)$.

Changing the ratio $k_{b}$ by (15) or (16) determine the alteration of the corresponding known power indicator: asymmetry coefficient by reverse sequence $K_{2}$, the power factor $\cos \varphi$, THD of voltage $T H D_{u}$ and current $T H D_{i}$. Fig. 4 illustrates the relationship of these power indicators with regularity of $k_{b}$ factor changes.

Fig. 5 shows the dependences of the correction factor $k_{l}$ on the $k_{b}$ factor which sets the pattern of changes of the asymmetrical operation mode of the three-phase three-wire ESS by (15). The values of the coefficient $k_{l}$ at $k_{b}=0$ and at $k_{b}=\sqrt{2}$ correspond to the three-phase ESS emergency operation mode at breakage of the line wire. The value of the coefficient $k_{l}$ at $k_{b}=1$ corresponds to the three-phase symmetrical operation at which the total power losses are equal to the lowest possible power losses.

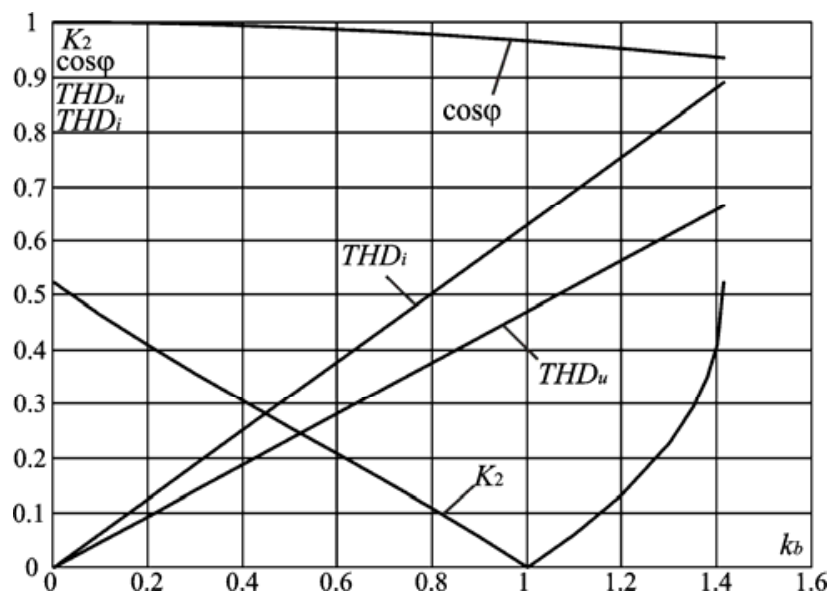

Fig. 4. Dependence of power indicators vs coefficient $k_{b}$ 


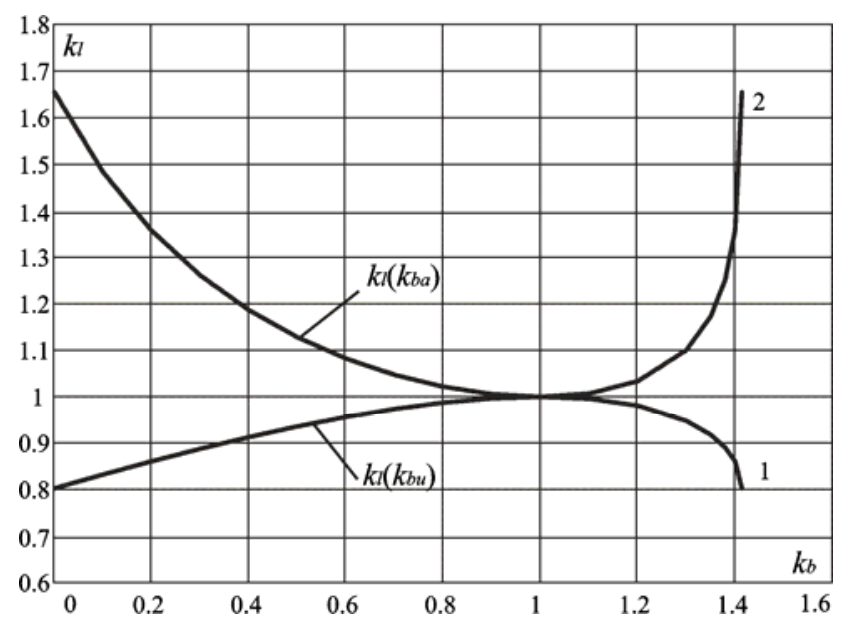

Fig. 5. Dependences $k_{l}=f\left(k_{b}\right)$ for three-phase ESS unbalanced modes: 1 - three-phase source unbalance; 2 - resistive load unbalance

Fig. 6 shows the dependence of the correction factor $k_{l}$ on the $k_{b}$ factor which sets the pattern of change of power factor and amplitudes of voltage and current higher harmonic of the three-phase four-wire ESS at $R_{n}=R_{s}$ by (16).

Additional power losses determination. Deviation of the operation conditions of the three-phase ESS from the conditions in which the power losses correspond to the minimum possible value, leads to additional losses [10]. Developed mathematical models allow us to calculate the value of power the additional losses in any of 279 modes of operation of the three-phase ESS. Fig. 7 shows the dependences of the relative, as a fraction of the average load useful power, power of additional losses on the $k_{b}$ factor for five considered ESS modes of operation.

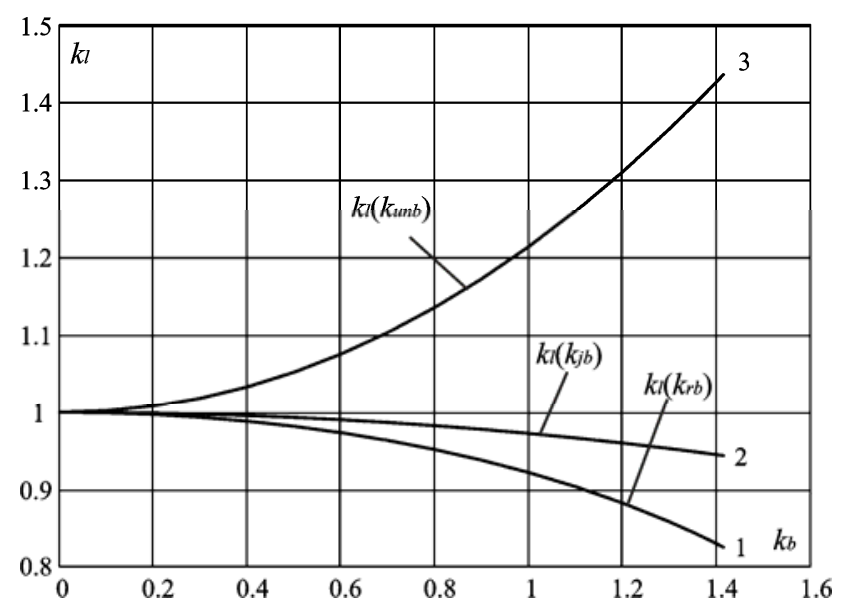

Fig. 6. Dependences $k_{l}=f\left(k_{b}\right)$ at: 1 - active-inductive load; $2-$ symmetric nonlinear load;

3 - three-phase voltage source higher harmonics
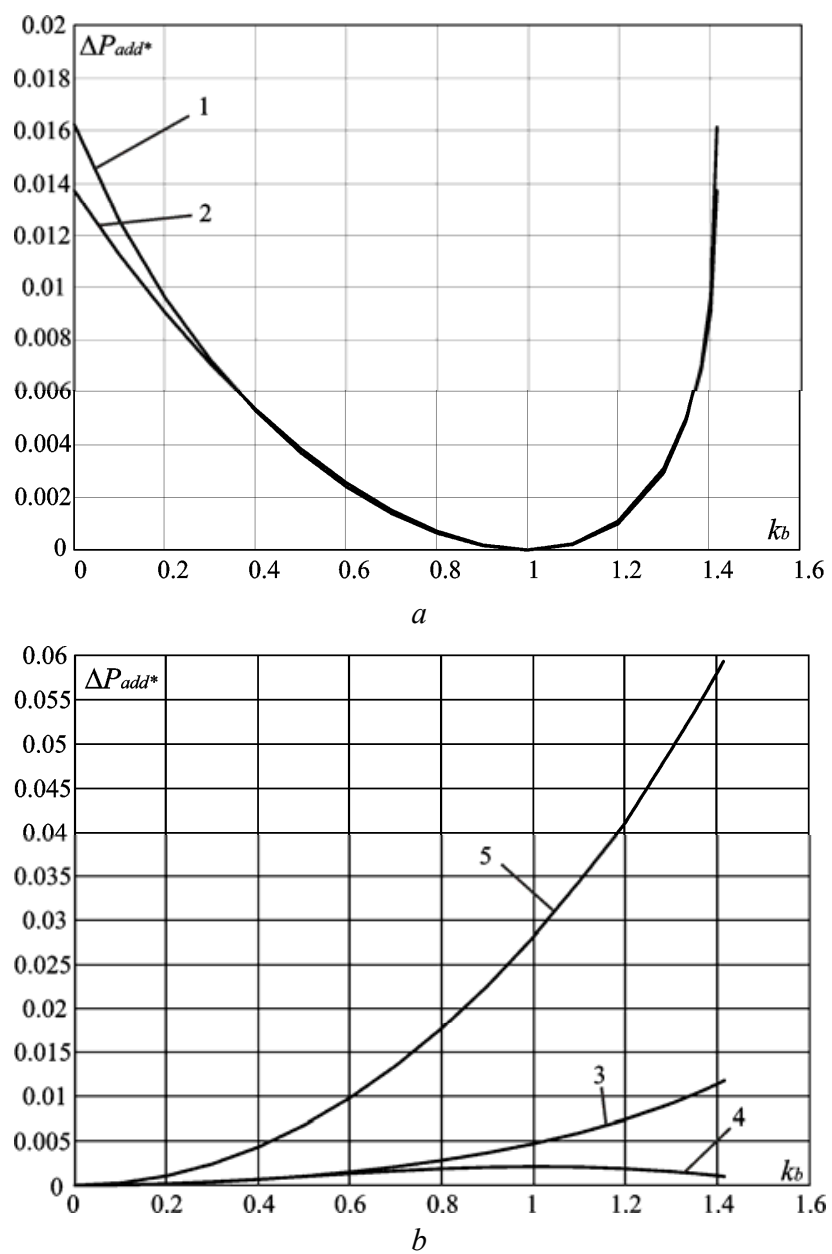

Fig. 7. Dependence of the relative additional power losses on the factor $k_{b}$

Analysis of the dependences in Fig. 7 shows that additional power losses in three-phase ESS at $k_{s c}=20$ can range from a few tenths to a few percent of the average load useful power. The three-phase ESS with non-linear load have the smallest power efficiency. A combination of factors causing additional losses decreases energy efficiency of three-phase ESS.

Analytical data obtained on the developed model (27) - (41) for five of considered cases are presented in Table 1. 
Calculation of correcting factors and power indicators for five operation modes of the three-phase ESS

\begin{tabular}{|c|c|c|c|c|c|c|c|c|c|c|c|c|c|c|c|}
\hline \multirow{2}{*}{$k_{b}$} & \multicolumn{3}{|c|}{ Mode 1} & \multicolumn{3}{|c|}{ Mode 2} & \multicolumn{3}{|c|}{ Mode 3} & \multicolumn{3}{|c|}{ Mode 4} & \multicolumn{3}{|c|}{ Mode 5} \\
\hline & $k_{l u}$ & $K_{2 \mathrm{U}}$ & $\Delta P_{a d d^{*}}$ & $k_{l a}$ & $K_{2 \mathrm{I}}$ & $\Delta P_{a d d^{*}}$ & $k_{l r}$ & $\mathrm{os} \varphi$ & $\Delta P_{a d d^{*}}$ & $k_{\text {lun }}$ & $T H D_{u}$ & $\Delta P_{a d d^{*}}$ & $k_{l j}$ & $T H D_{i}$ & $P_{a d d}$ \\
\hline 0 & .8028 & 0.522 & 7 & 6551 & 522 & 2 & 1 & & & 1 & 0 & ) & 1 & 0 & 0 \\
\hline 0.1 & 324 & 462 & z & 839 & 462 & 1 & ( & 1 & 4 & 1.0021 & 0.047 & 0.00005 & 9997 & 0.041 & 0.000 \\
\hline 0.2 & 000 & 07 & & 570 & 407 & & 9971 & 999 & 16 & 4 & 094 & .0002 & 0.9989 & .082 & 001 \\
\hline 0 . & 877 & 354 & & 615 & 354 & 2 & 935 & 997 & 7 & 38 & 141 & 04 & 75 & 0.123 & .002 \\
\hline 0.4 & 9129 & 304 & 3 & 1868 & 0.304 & 53 & 883 & 994 & 0.0 & 36 & 188 & 0.0007 & 0.9957 & 0.164 & 002 \\
\hline 0.5 & 0.9359 & 254 & 0.0038 & 1.1282 & 254 & & 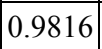 & 1 & 4 & & 0.235 & 01 & 32 & 0.205 & 0006 \\
\hline 0.6 & 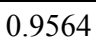 & 06 & & 7 & 6 & & 33 & 37 & 0.0 & & 82 & & & 6 & 00 \\
\hline 0 . & 738 & 157 & 5 & 473 & 157 & 4 & 634 & 0.983 & 0.00212 & 037 & 329 & 0.00164 & 0.9866 & .287 & 0.013 \\
\hline 0.8 & 9875 & 0.107 & 07 & 1.0218 & 0.107 & 964 & 0.9516 & 0.978 & 0.00284 & 1.1359 & 0.367 & 0.0019 & 0.9825 & 0.328 & 0.017 \\
\hline 0.9 & 9966 & 0.055 & 0002 & .0058 & 0.055 & 00017 & 378 & 972 & 0.0 & 1.1726 & 0.423 & 0.0021 & 0.9778 & 0.368 & 0.022 \\
\hline 1.0 & 1 & 0 & 0 & 1 & 0 & 0 & 19 & 56 & 0.0047 & 14 & 0.47 & 13 & 0 & 99 & 0.020 \\
\hline 1.1 & 9958 & 0.061 & 00023 & 1.0071 & 0.061 & 21 & 0.9035 & 0.959 & 0.00595 & 1.26 & 0.517 & 0.0021 & 0.9665 & 0.45 & 0.032 \\
\hline 1.2 & 0.9808 & 0.133 & 11 & 1.0338 & 0.133 & 01 & 0.8825 & .952 & 0.00743 & 1.3109 & 0.564 & 0.0019 & 0.96 & 0.491 & 0.041 \\
\hline 1.3 & 79 & 27 & 0.0031 & 8 & 27 & & 1 & 4 & 0.0 & 55 & 611 & 66 & 25 & 32 & 0040 \\
\hline 1.35 & 18 & 293 & 0.0049 & 81 & 0.293 & 9 & 3 & 0.94 & 0.01 & 1.3962 & 0.634 & 0.0 & 19 & 553 & 0.0 \\
\hline 1.38 & 0.8901 & 0.35 & 69 & 1.2538 & 0.35 & 7 & 358 & 938 & 0.01095 & 1.4145 & 0.648 & 0.0012 & 0.9466 & 0.565 & 0.056 \\
\hline 1.4 & 8 & 0 & 0 & 1.3576 & 0 & 96 & 8 & 6 & 4 & 27 & 7 & 1 & 15 & 73 & 0.058 \\
\hline .414 & 28 & 22 & 37 & 51 & 2 & 61 & 54 & 5 & 0.0118 & 6 & 54 & 0.00101 & 8 & 79 & ועם \\
\hline
\end{tabular}

Three-phase ESS Matlab-model. In accordance with the equivalent circuit of Fig. 2 and matrix mathematical model (27) - (41) a Matlab-model of the three-phase ESS shown in Fig. 8 was created. It differs from the previously developed models $[5,6,11]$ by the presence of optimized load structure which is divided into a symmetric resistive load and a adjustable symmetrical three-phase current source and a block of automated calculation of the correction factor $k_{l}$ ( $\mathrm{kl}$ Calculation). The model allows at setting the values of the elements of the vectors (8) - (12) without an operator to automatically calculate the coefficient $k_{l}$ and calculate the values of the total power losses components taking into account the calculated ratio.

The Matlab-model consists of:

1. the power circuit configured by Fig. 2 and included:

- the three-phase source of symmetric sinusoidal voltages Usa, Usb, Usc;

- the three-phase source of non-sinusoidal voltages $\mathrm{Ua}, \mathrm{Ub}, \mathrm{Uc}$;

- the symmetrical resistive load Rla, Rlb, Rlc;

- the three-phase current source $\mathrm{Ja}, \mathrm{Jb}, \mathrm{Jc}$;

- the active resistances of the four-wire line $\mathrm{Ra}, \mathrm{Rb}$, Rc, Rn;

- the power active filter configured on three adjustable current sources SAF.

2. the current and voltage sensors connected to the model's power circuit.

3. the subsystems of the measured data processing:

- the subsystem of the total power losses components calculation by (1) Calculation 1 (Fig. 9);

- the Subsystem of the total power losses components calculation in p-q-r coordinates Calculation 2 (Fig. 10);

- the subsystem of load useful power harmonic composition calculation Calculation 3;

- the subsystem of the total power losses components calculation by coefficients of direct, reverse and zero consequences Calculation 4 (Fig. 11);

- the subsystem of calculation and indication of measured information on instantaneous values of electrical quantities and three-phase ESS parameters Measurements 1;

- the subsystem of calculation and indication of measured information on average and RMS values of electrical quantities and three-phase ESS parameters Measurements 2 (Fig. 12).

4. the subsystems of the ESS state control and monitoring:

- the PAF control system SAF Controller (Fig. 13);

- the subsystem for setting control signals for the three-phase current source and three-phase non-sinusoidal voltage source Control System;

- the subsystem of the correcting factor $k_{l}$ calculation kl Calculation;

- the block for the PAF connection to the ESS PAF Connection.

5. the virtual measured devices.

The developed Matlab-model can be used to analyze the three-phase energy supply systems and for assessment the energy efficiency at to a power system of the active power filter at any of 288 possible modes of operation. 


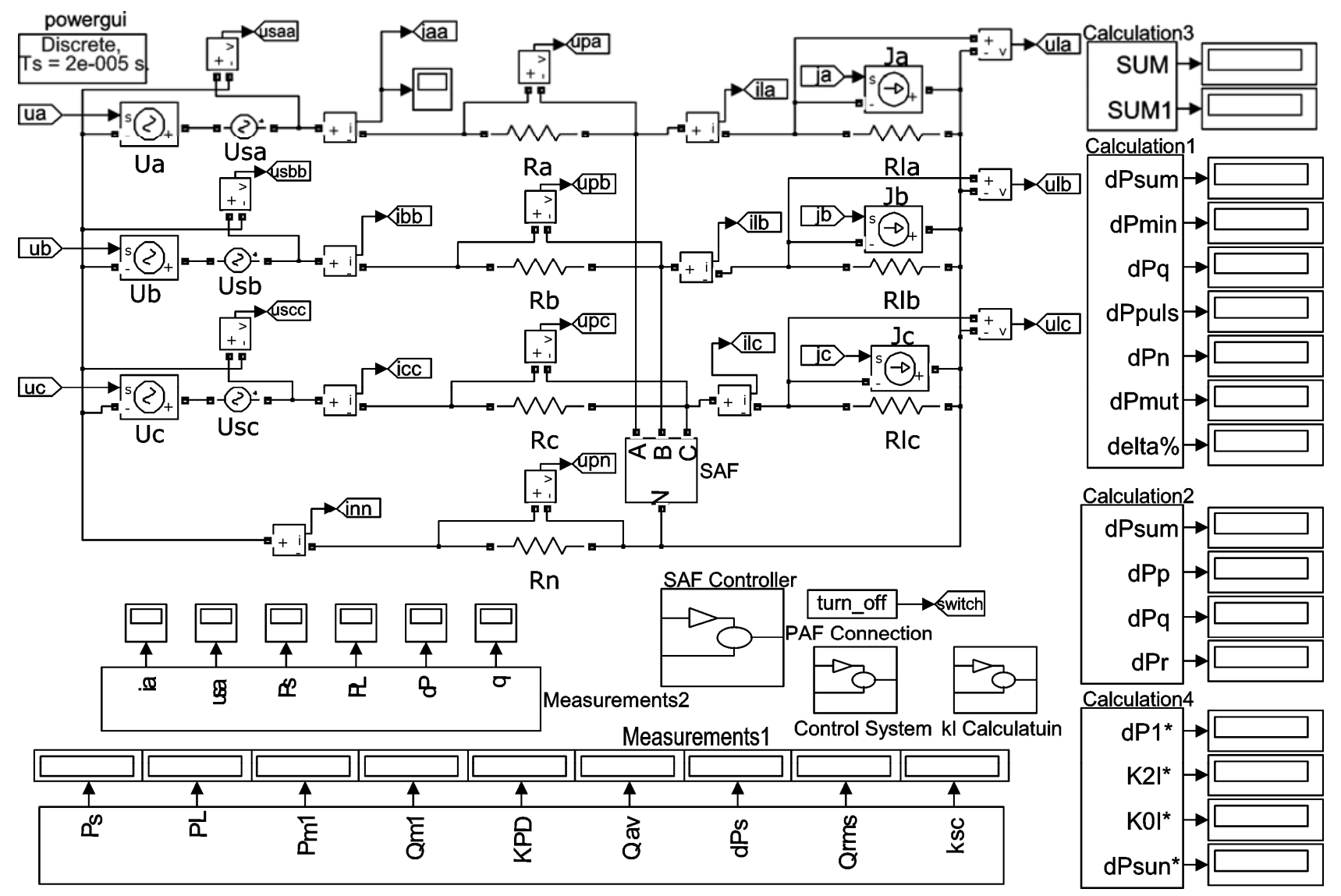

Fig. 8. Matlab-model of the three-phase ESS with PAF

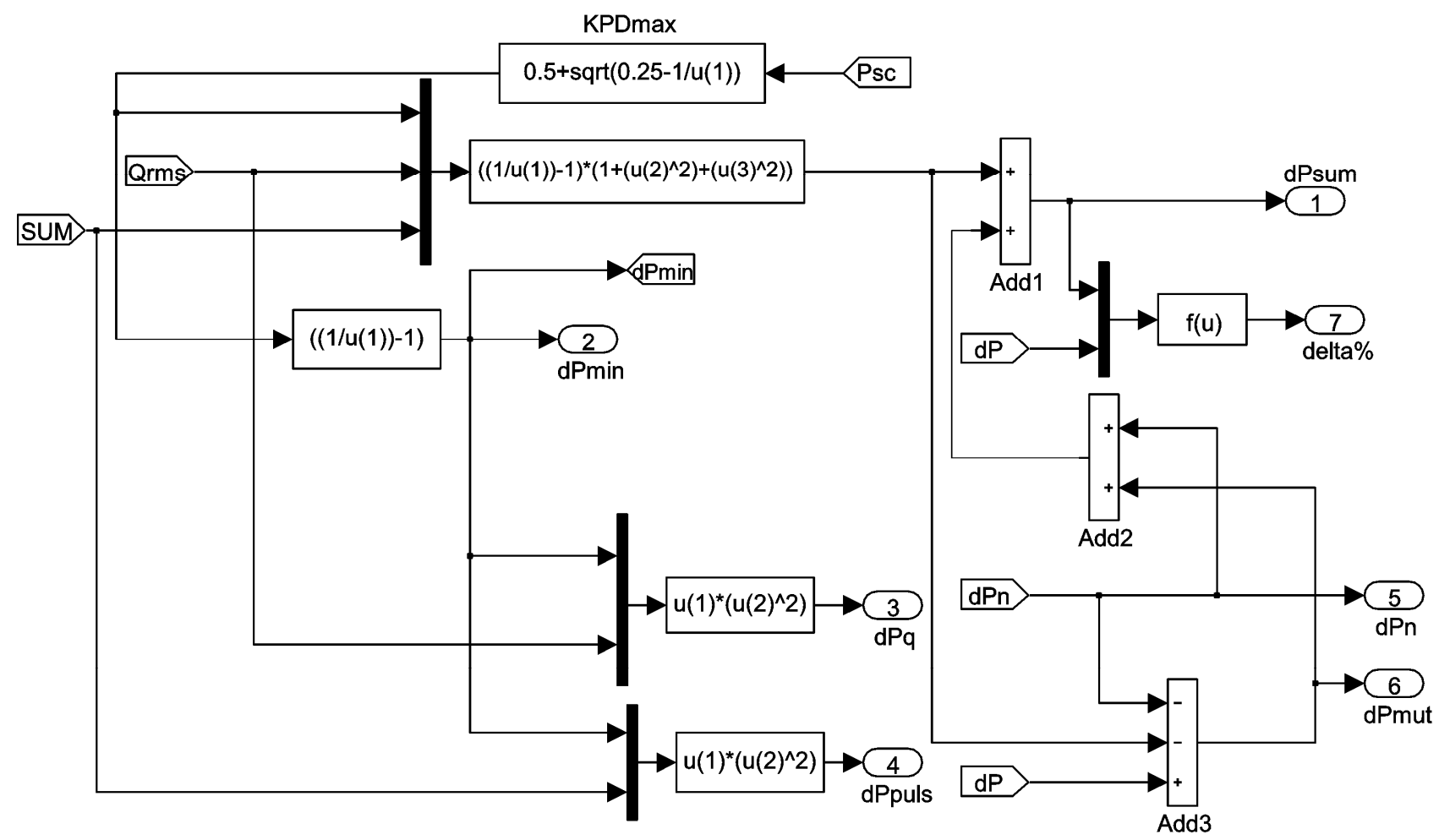

Fig. 9. Subsystem of the total power losses components calculation by (1) Calculation 1 


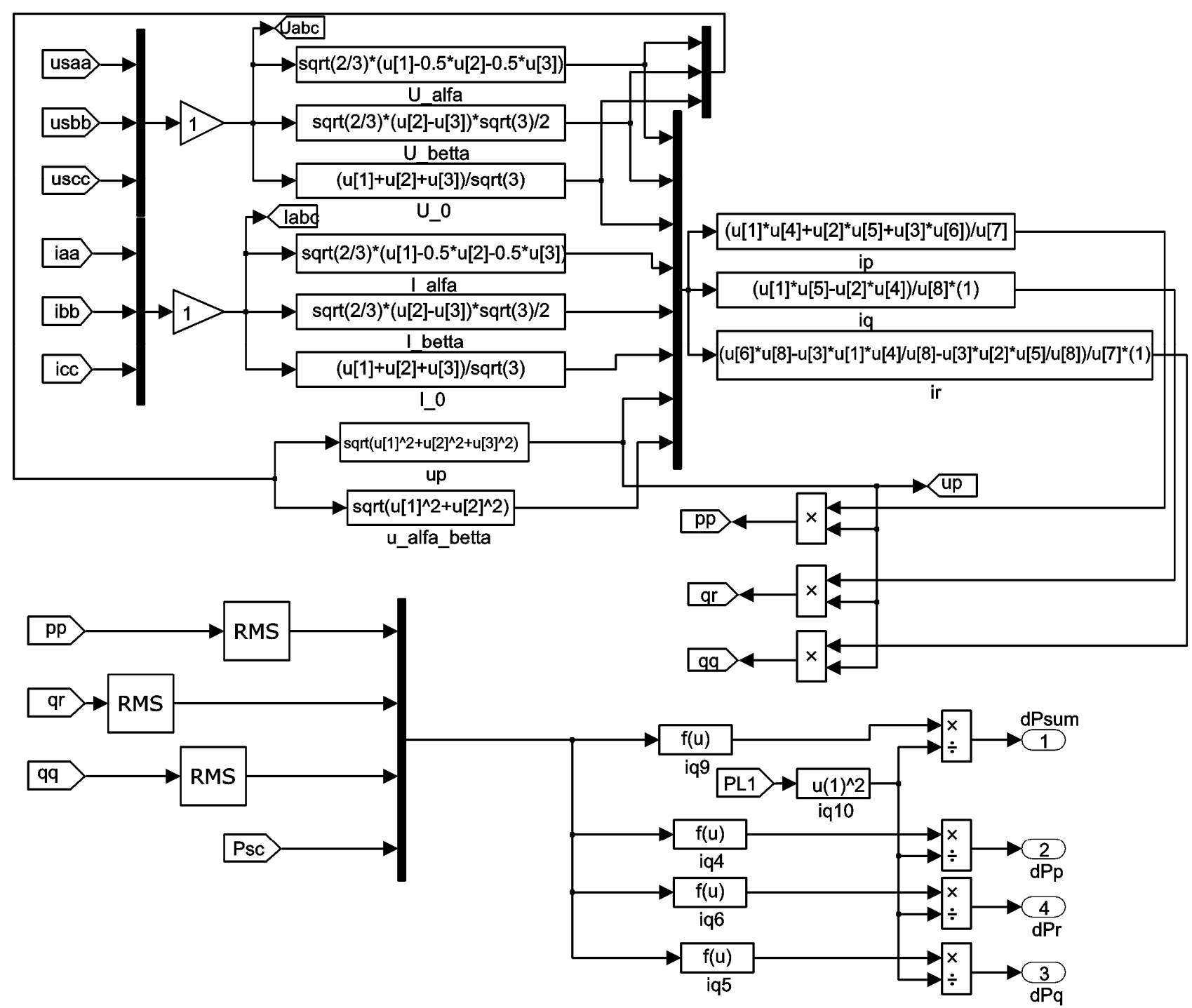

Fig. 10. Subsystem of the total power losses components calculation in p-q-r coordinates Calculation 2

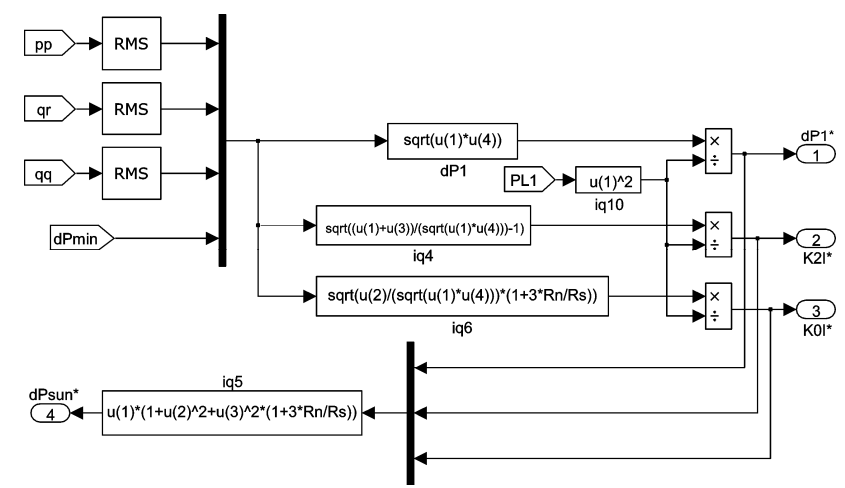

Fig. 11. Subsystem of the total power losses components calculation by coefficients of direct, reverse and zero consequences Calculation 4

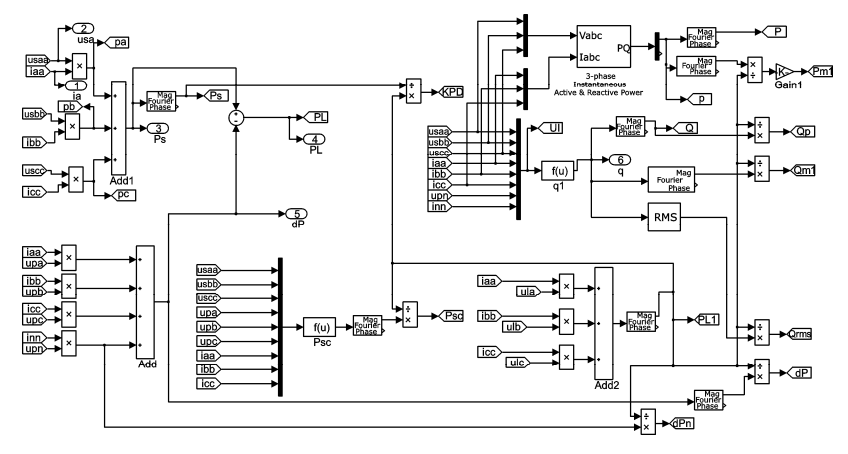

Fig. 12. Subsystem of calculation and indication of measured information on average and RMS values of electrical quantities and three-phase ESS parameters Measurements 2 


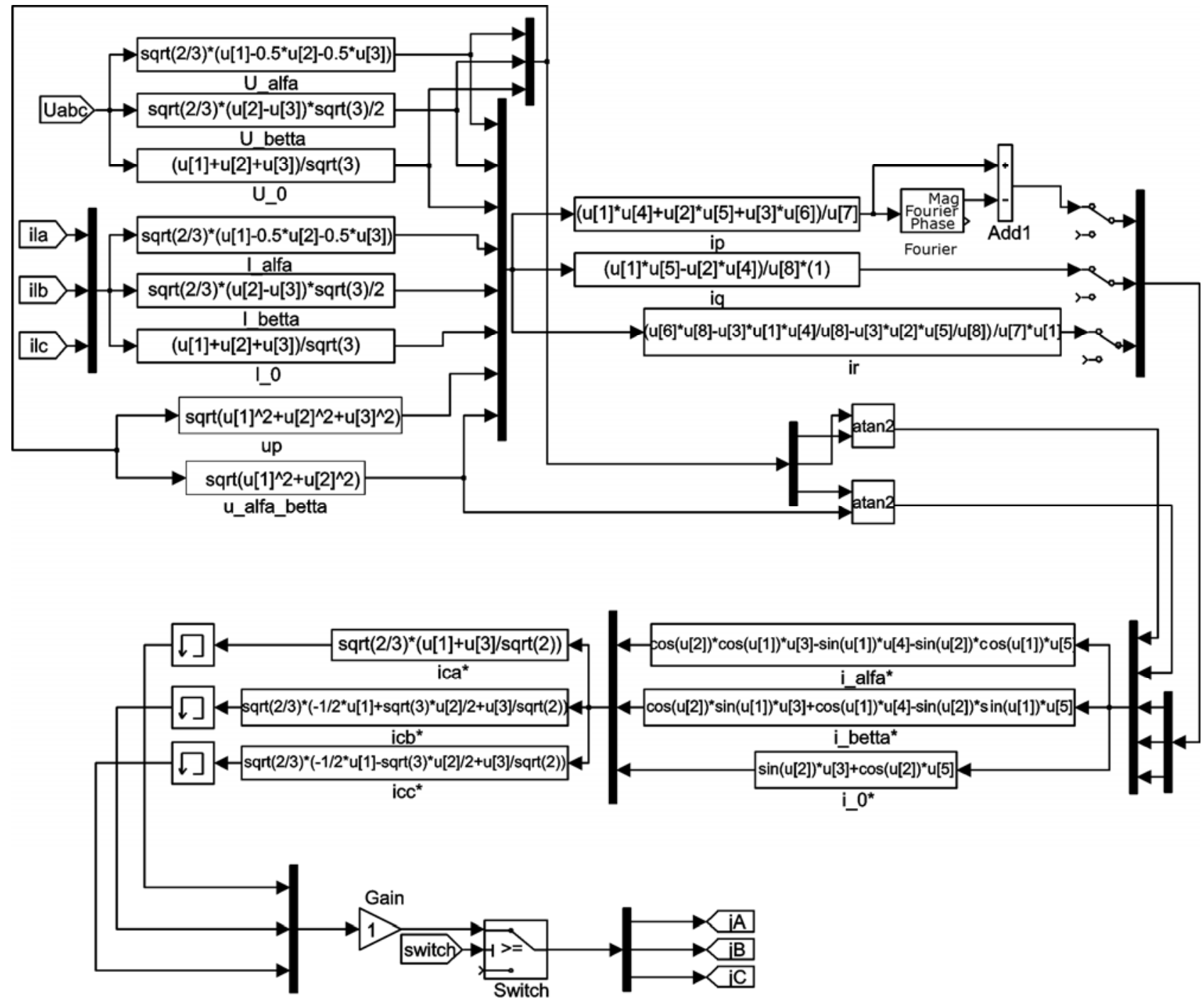

Fig. 13. PAF control system SAF Controller

\section{Conclusions.}

1. We proposed two ways to create a mathematical model of the three-phase ESS, the first one is based on the vector form, and the second one is based on the matrix representation of power processes in multiphase systems. It allows solving the problem of maintaining of constant values of average active load power in the ESS operation at different modes.

2. Based on the analysis of the results of modeling of five operating modes of the three-phase ESS, at each of which a unique factor that causes the appearance of additional losses in the system is involved, it was concluded that the maximum reduction of power efficiency corresponds the ESS with non-linear load.

3. Using the developed mathematical models the operation of the MATLAB-model of the three-phase ESS with PAF is optimized. The possibility of the automated calculation of the correction factor $k_{l}$ is realized which is more than an order reduced the working time with model at investigation of the ESS operating modes at which additional losses arise

\section{REFERENCES}

1. Akagi H., Kanazawa Y., Nabae A. Instantaneous reactive power compensators comprising switching devices without energy storage components. IEEE Transactions on Industry Applications, 1984, vol.IA-20, no.3, pp. 625-630. doi: 10.1109/TIA.1984.4504460.

2. Wong M.-C., Dai N.-Y., Lam C.-S. «Active Power Filters», Parallel Power Electronics Filters in Three-Phase Four-Wire Systems. Springer Singapore, 2016. pp. 59-165. doi: 10.1007/978-981-10-1530-4_3.

3. Artemenko M.Yu., Batrak L.M., Mykhalskyi V.M., Polishchuk S.Y. Analysis of possibility to increase the efficiency of three-phase four-wire power system by means of shunt active filter. Tekhnichna elektrodynamika, 2015, no.6, pp. 12-18. (Ukr). 4. Artemenko M.Yu., Batrak L.M., Polishchuk S.Y., Mykhalskyi V.M., Shapoval I.A. The effect of load power factor on the efficiency of three-phase four-wire power system with shunt active filter. 2016 IEEE 36th International Conference on Electronics and Nanotechnology (ELNANO), IEEE, 2016. pp. 277-282. doi: 10.1109/ELNANO.2016.7493067.

5. Zhemerov G.G., Tugay D.V. Physical meaning of the «reactive power» concept applied to three-phase energy supply systems with non-linear load. Electrical engineering \& electromechanics, 2015, no.6, pp. 36-42. (Rus). doi: 10.20998/2074-272X.2015.6.06. 
6. Zhemerov G.G., Tugay D.V. An universal formula clarification to determine the power losses in the three-phase energy supply systems. Bulletin of NTU «KhPI», 2015, no.12, pp. 339-343. (Rus).

7. Peng F.Z., Ott G.W., Adams D.J. Harmonic and reactive power compensation based on the generalized instantaneous reactive power theory for three-phase four-wire systems. IEEE Transactions on Power Electronics, 1998, vol.13, no.6, pp. 1174-1181. doi: 10.1109/63.728344.

8. Afonso J., Couto C., Martins J. Active filters with control based on $\mathrm{p}$-q theory. IEEE Industrial Electronics Society Newsletter, 2000, vol.47, no.3, pp. 5-10.

9. Kim H.S., Akagi H. The instantaneous power theory on the rotating p-q-r reference frames. Proceedings of the IEEE 1999 International Conference on Power Electronics and Drive Systems. PEDS'99 (Cat. No.99TH8475), 1999, pp. 422-427. doi: 10.1109/PEDS.1999.794600.

10. G. Zhemerov, N. Ilina, D. Tugay. The Theorem of Minimum Energy Losses in Three-Phase Four-Wire Energy Supply System. 2016 2nd IEEE International Conference on
Intelligent Energy and Power Systems (IEPS-2016). June 07-11, 2016, Kyiv, Ukraine, pp. 52-54. doi: 10.1109/IEPS.2016.7521889.

11. Zhemerov G.G., Tugay D.V. Components of total electric energy losses power in pqr spatial coordinates. Electrical engineering \& electromechanics, 2016, no.2, pp. 11-19. (Rus). doi: 10.20998/2074-272X.2016.2.02.

Received 01.06.2016

D.V. Tugay, Candidate of Technical Science, Associate Professor

O.M. Beketov National University of Urban Economy in Kharkiv,

12, Revolution Str., Kharkiv, 61002, Ukraine.

phone+38057 7073111, e-mail: tugaydv@yandex.ua

How to cite this article:

Tugay D.V. Three-phase energy supply systems simulation for the total power losses components assessment. Electrical engineering \& electromechanics, 2016, no.4, pp. 43-53. doi: 10.20998/2074-272X.2016.4.06. 